\title{
Análisis del escenario de la certificación forestal en el contexto del desarrollo sustentable
}

\author{
Juan Carlos Tamarit Urias ${ }^{1}$
}

\begin{abstract}
RESUMEN
La certificación forestal es un instrumento económico basado en el mercado que tiene como objetivo fomentar el manejo forestal sustentable mediante el acceso al mercado y un aumento en el precio de la madera que permitirá cubrir los costos del buen manejo del bosque. Mediante el análisis de los costos por certificación y comportamiento del mercado de la madera certificada, en este trabajo se determina la efectividad que la certificación forestal ha tenido sobre las dimensiones social, económica y ambiental así como su contribución al manejo forestal sustentable. Se concluyó que los costos de certificación son altos para pequeños productores y empresas de países en desarrollo. El mercado de la madera certificada es marginal, su demanda y oferta limitadas y el sobreprecio incierto, no contribuyendo al desarrollo socioeconómico de comunidades situadas en bosques degradados con especies de bajo valor comercial, por lo que la pobreza, degradación y deforestación en esas zonas continúa presente. La certificación forestal sólo considera a la madera como producto único del bosque con valor en el mercado; los productos forestales no maderables y los bienes y servicios ambientales no son considerados puesto que se carece de estándares aplicables a éstos, subestimándose el valor real de los bosques. Estas limitaciones llevan el riesgo de desmotivar el manejo forestal, inducir al aprovechamiento ilegal y a cambios de uso del suelo. La certificación forestal sólo contribuye parcialmente al manejo forestal sustentable y únicamente se concibe como una herramienta alternativa que se encuentra en pleno desarrollo.
\end{abstract}

PALABRAS CLAVE:

Certificación forestal, desarrollo sustentable, ecosistemas forestales, costos de certificación, mercado de madera certificada.

\begin{abstract}
Forest certification is an economic instrument based on the market which has as objective to promote the sustainable forest management by means of the access to the market as well as an increase in the price of the wood what will allow to cover the costs of the good forest management. In this work it is analyzed the impact and the effectiveness that forest certification has had on the social, economic and environmental dimensions on which is based and its contribution to the sustainable forest management, analyzing its costs and behavior of the market of certified wood. It was concluded that the certification costs are high for small producers and companies in developing countries. The market of certified wood is marginal, demand and offer are limited, the increase in the price is uncertain and far from clear, it does not contribute to the socioeconomic development of communities located in forests degraded with tree species of low commercial value with poverty, degradation and deforestation being still present in those areas. Forest certification considers the wood as the only product obtained from the forest with market value, non-wood forest products along with environmental goods and services are not considered as commercially valuables due to the lack of standards applicable to these, underestimating the actual value of the forests. These limitations carry the risk discouraging the forest management and inducing the illegal logging, and changing the use of forest land. Forest certification contributes partially to the sustainable forest management and it is, only considered as an alternative tool, which is still being developed.
\end{abstract}

KEY WORDS:

Forest certification, sustainable development, forests ecosystems, costs of the certification, certified wood market. 


\section{INTRODUCCIÓN}

El concepto manejo forestal sustentable (MFS) surge por la degradación evidente de los recursos forestales tanto en cantidad como en calidad. Su definición comprende aspectos económicos, ambientales y socioculturales. Rametsteiner (2001) indica que en las próximas décadas será el enfoque preponderante, puesto que es íntegro y holístico. De acuerdo con Savenije (1999), el MFS es un concepto genérico y dinámico que requiere definiciones precisas por lo que las formas de medirlo están en pleno desarrollo y evolución, no obstante, la premisa básica del desarrollo sustentable perdura.

El MFS consiste en manejar los bosques como ecosistemas y no como simples productores de madera (Aguirre et al., 2002; FAO, 2003), los componentes bióticos y abióticos del ecosistema forestal tienen interrelaciones complejas que deben ser suficientemente comprendidas (Savenije, 1999). El MFS también debe satisfacer las necesidades económicas, sociales y ambientales de las generaciones presentes y futuras, para ello deben identificarse y clasificarse las funciones de los bosques en: reguladoras, de soporte, productivas, de información, turísticas, etc.

Savenije (1999) señala que cada bosque puede mantener su capacidad de soporte productiva y de regeneración solo sí se maneja como ecosistema, Salim et al. (1997) agrega que se considera sustentable si retiene íntegros sus componentes bióticos y los procesos ecológicos a través del tiempo, manteniéndose con ello sus funciones económicas, ecológicas y sociales.

Para frenar y revertir el problema de degradación y deforestación de los bosques se han desarrollado diversas estrategias y acciones tanto nacionales como internacionales que no han dado los resultados esperados principalmente en los países en desarrollo. En la mayoría de ellos, la deforestación es alta y ha contribuido significativamente que, a nivel mundial, en la década 1990-2000 la tasa anual de deforestación fuera de 14,6 millones de hectáreas (van Dam, 2002; FAO, 2001; FAO, 2003).

Una de las estrategias para atender esta problemática es la certificación forestal cuyo planteamiento es fomentar el buen manejo forestal que sea socialmente beneficioso, ambientalmente responsable y económicamente viable (Upton y Bass, 1995; Rice et al., 2001).

Los principales sistemas internacionales de certificación son: el Pan Europeo (Pan European Forest Certification o PEFC); el Consejo de Administración Forestal (Forest Stewardship Council o FSC), la Organización Internacional de Normalización (International Organization Standarization o ISO) en su serie ISO-14001, además hay otros sistemas nacionales que recientemente algunos países han desarrollado (FAO, 2001; Sasser, 2002).

La certificación forestal es un instrumento económico basado en el mercado (Aguirre et al., 2000; Rice et al., 2001; van Dam, 2002), tiene entre sus objetivos proporcionar un incentivo de mercado para mejorar la gestión forestal y fomentar efectos favorables sobre los ecosistemas forestales a través del cambio de comportamientos en los consumidores (Salim et al., 1997; Rametsteiner, 2001).

Hasta ahora la certificación forestal es voluntaria. Cuando se lleva a cabo, implícitamente comprende un MFS que es evaluado por una empresa ajena a la certificadora y al propietario del bosque. La empresa evaluadora emplea un enfoque normativo en el que se verifican principios, criterios e indicadores que conforman un estándar reconocido y compatible internacionalmente.

La certificación forestal se puede aplicar a: todo tipo de bosques (tropical, 
templado y boreal), todas las modalidades de tenencia de la tierra, diferentes tamaños de predio o unidades de manejo forestal (UMF) y bajo condiciones socioeconómicas variadas (Barthod, 1998; Savenije, 1999; Simula, 2001).

La certificación forestal considera también la certificación de los productos mediante cadenas de custodia. En ellas hay un seguimiento de la madera en todos los procesos de transformación hasta que el producto llega al consumidor final. Inicialmente la certificación forestal fue impulsada por organizaciones no gubernamentales ambientales y actualmente también es promovida por empresas e industrias que buscan asegurar el mercado para sus productos (Rametsteiner, 2001; Simula, 2001).

En años recientes la certificación forestal ha formado parte de la agenda ambiental, económica y política internacional, siendo objeto de una atención creciente en diversos foros, como la Unión Europea, la FAO, la Comisión para el Desarrollo Sustentable de las Naciones Unidas, entre otras (Kiekens, 1995). Aún cuando es un instrumento nuevo en pleno desarrollo, a una década de que surgió a nivel mundial se han certificado 109 millones de hectáreas de bosques, que representa el 2,5\% del área forestal mundial (van Dam, 2002).

El presente trabajo es producto de una investigación documental en la que se revisó literatura especializada de actualidad sobre el desarrollo de la certificación forestal y su impacto en el manejo forestal sustentable. Se parte del análisis tanto de los costos por lograr la certificación como de la superficie certificada, además, se definen las características del mercado de la madera certificada así como las principales limitaciones de la certificación forestal. Para el análisis y discusión se parte de la situación que prevalece en el ámbito internacional para después realizar algunas precisiones y particularidades para las condiciones específicas de México.

\section{OBJETIVO}

Determinar, en el ámbito internacional con algunas precisiones para el caso de México, la efectividad que la certificación forestal tiene sobre las dimensiones social, económica y ambiental, así como su contribución al manejo forestal sustentable a través del análisis de los costos por certificación y del mercado de la madera certificada.

\section{COSTOS DE LA CERTIFICACIÓN}

El costo que implica el proceso de certificación está en función de la superficie y tipo de bosque (natural 0 plantación), de la diferencia entre el estándar a ser utilizado en la evaluación y el estado actual del manejo forestal (Simula, 2001). Muchos gastos pueden reducirse y aún evitarse cuando el manejo forestal realizado está cercano a los estándares de certificación (Fig. 1); así, un sistema de manejo, administración y prácticas forestales adecuadas constituyen la base necesaria para ejecutar la certificación en una forma práctica y efectiva en costos (Savenije, 1999).

Hay costos directos e indirectos, los primeros son realizados para cubrir la operación de la certificación y se pagan a la organización certificadora. Los costos indirectos son lo que se realizan en el manejo forestal y los beneficios que se pierden (costos de oportunidad) si no se realizara la certificación. En muchos casos, los costos indirectos son mayores que los directos; el costo total de la certificación varía dependiendo de las condiciones locales de cada región o país. Todos los costos derivados por la certificación y por realizar un manejo sustentable son absorbidos exclusivamente por el productor forestal y no son transferidos al consumidor (van Dam, 2002). 


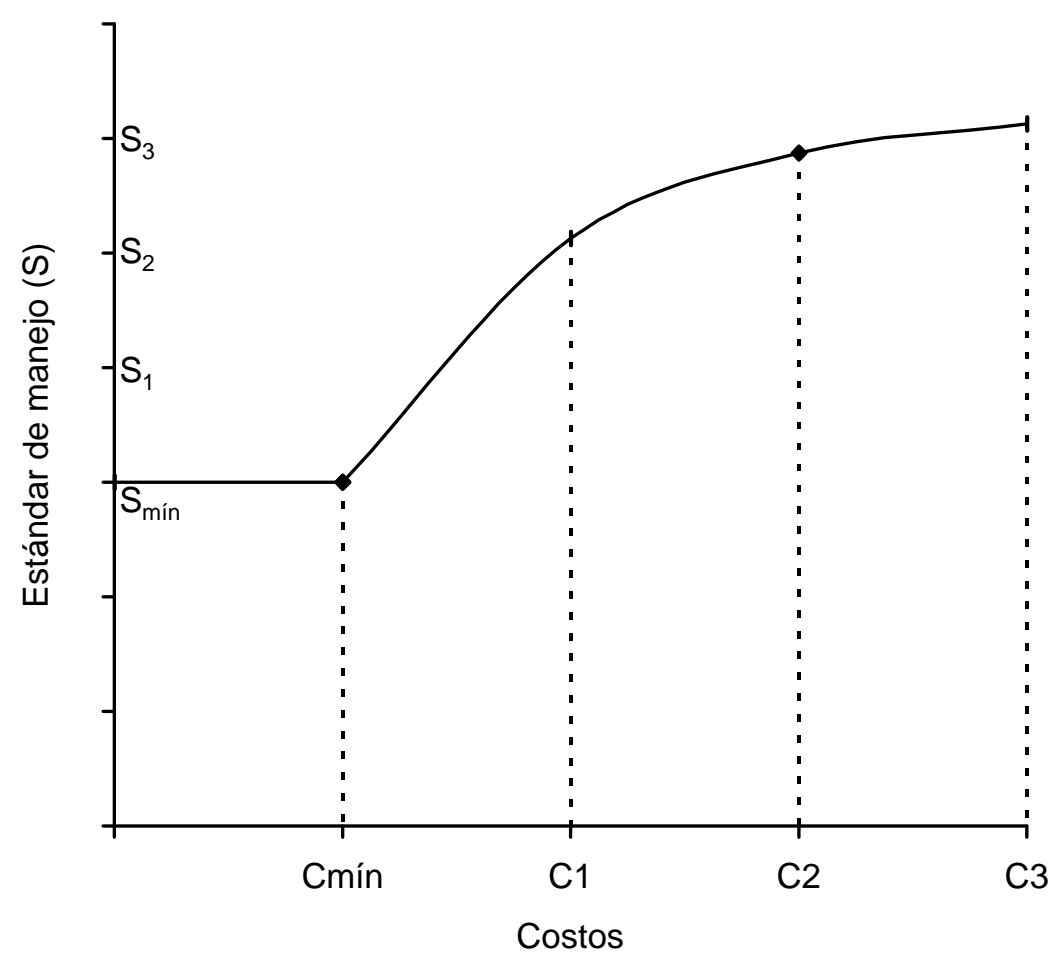

Figura 1. Costos de certificación en función del estándar de manejo.

Otro problema importante es que los costos por conservar los bienes y servicios no económicos del bosque como regulación del clima, mejoramiento de la calidad del aire, conservación del suelo, captación de agua, conservación del paisaje y biodiversidad, etc., son difíciles de evaluar monetariamente y también los absorbe totalmente el productor forestal, en tanto que los beneficios los recibe toda la sociedad (Baharuddin, 1995).

Bajo estas condiciones, la certificación se convierte en exclusiva de grandes productores y empresas de países desarrollados cuyo nivel de producción y rentabilidad les permiten pagar los costos del proceso. Así, los pequeños productores y las empresas medianas de países en desarrollo no están en condiciones de certificarse sin altos costos. Además carecen de tecnologías y capital por lo que resultan mayores los costos unitarios durante el proceso de evaluación, menores ingresos y una repartición desincronizada de gastos y beneficios en el tiempo.

Los pequeños productores y las empresas medianas referidas, con mucho esfuerzo logran certificarse con un alto número de condicionantes, observaciones y recomendaciones, que combinadas con una administración deficiente, unas baja capacidad de negociación para obtener un sobreprecio de su madera, una baja productividad, una infraestructura obsoleta y un limitado acceso a la información los lleva a perder la certificación en evaluaciones posteriores.

En general el costo de la certificación se incrementa a medida que la superficie de bosques a certificar disminuye (Tabla 1). En México la certificación de una superficie menor a 1000 ha no es rentable (van Dam, 2002), razón por la que los productores utilizan el esquema 
de "paraguas" o en grupos para certificarse. Esto, aún cuando permite reducir el costo a una décima parte del precio normal de una certificación forestal individual (Tickell, 2000), sigue siendo alto y los productores no pueden amortizarlo.

\section{SUPERFICIE CERTIFICADA}

La superficie certificada a escala mundial está desigualmente distribuida. De un total de 109 millones de hectáreas, más del $50 \%$ se localiza en Europa, el 40 $\%$ en USA y Canadá y sólo el $8 \%$ está en países en desarrollo de África, América Latina y Asia-Pacífico (van Dam, 2002). Es claro que se han beneficiado los países desarrollados, las grandes empresas y los bosques templados y boreales antes que los tropicales, caracterizados por ser centros únicos y frágiles de biodiversidad y en ellos las prácticas de aprovechamiento en general no son sustentables.

En América Latina todos los bosques y plantaciones certificados están bajo el esquema FSC (Tabla 2), la superficie certificada de 3,66 millones de hectáreas representa el $9,88 \%$ del total. Sin considerar a México y Guatemala, más del $97 \%$ de las certificaciones está en manos

Tabla 1. Costos de certificación en América Latina

\begin{tabular}{cc}
\hline $\begin{array}{c}\text { SUPERFICIE A } \\
\text { CERTIFICAR (ha) }\end{array}$ & $\begin{array}{c}\text { COSTO } \\
(\mathrm{U} \$ / \mathrm{ha})\end{array}$ \\
\hline 750 & 21,3 \\
\hline 1500 & 12,0 \\
\hline 6300 & 8,7 \\
\hline 14900 & 2,6 \\
\hline 25000 & 0,8 \\
\hline 80000 & 0,6 \\
\hline
\end{tabular}

de grandes productores y empresas privadas. De la superficie mundial certificada, los bosques comunales certificados comprenden el $3 \%$; considerando solo a América Latina, estos bosques ocupan el $23,5 \%$ de la superficie certificada. De esta superficie comunal el $85 \%$ está en México y Guatemala, países en los que las reformas a las leyes forestales y los cambios en las políticas públicas del sector forestal en los últimos años aunados a la cooperación internacional y la buena organización de las comunidades ha favorecido el proceso.

En el caso específico de México, la superficie certificada corresponde en un $94 \%$ a bosques comunales y ejidales, situación normal considerando que prácticamente el $80 \%$ de los bosques se encuentra bajo el régimen de tenencia comunal y ejidal. Sin embargo, la superficie certificada representa solamente el $6,4 \%$ de los bosques bajo manejo y el $2,5 \%$ de la superficie con potencial para la producción comercial maderable.

\section{EL MERCADO Y LOS PRECIOS DE LA MADERA CERTIFICADA}

En teoría, la certificación debe mejorar el manejo forestal mediante el acceso seguro al mercado internacional de la madera y sus productos derivados. Esto garantizaría cubrir los costos que conlleva el buen manejo, a la vez que se tendría un incremento de los precios (Baharuddin, 1995; Cabarle y de Freitas, 1995; Salim et al., 1997; Savenije, 1999). Sin embargo, el beneficio principal ha sido facilitar el acceso a los mercados sin un aumento significativo de los precios (Castilleja, 1993; Simula, 2001).

La certificación forestal no constituye ninguna garantía ni de mercado ni de precio. Si el sobreprecio no existe, es pequeño o incierto, la rentabilidad será 
Tabla 2. Superficie certificada por el FSC en América Latina por tipo de propiedad y bosque

\begin{tabular}{|c|c|c|c|c|c|c|c|c|c|}
\hline \multirow[b]{3}{*}{ País } & \multicolumn{4}{|c|}{ PRIVADA } & \multicolumn{2}{|c|}{ COMUNAL } & \multirow{2}{*}{\multicolumn{2}{|c|}{ Total }} & \multirow{3}{*}{ nco* } \\
\hline & \multicolumn{2}{|c|}{$\begin{array}{l}\text { BOSQUE } \\
\text { NATURAL }\end{array}$} & \multicolumn{2}{|c|}{ PLANTACIÓN } & \multicolumn{2}{|c|}{$\begin{array}{l}\text { BOSQUE } \\
\text { NATURAL }\end{array}$} & & & \\
\hline & $\begin{array}{c}<10000 \\
\text { ha }\end{array}$ & $\begin{array}{c}>10000 \\
\text { ha }\end{array}$ & $\begin{array}{c}<10000 \\
\text { ha }\end{array}$ & $\begin{array}{c}>10000 \\
\text { ha }\end{array}$ & $\begin{array}{c}<10000 \\
\text { ha }\end{array}$ & $\begin{array}{c}>10000 \\
\text { ha }\end{array}$ & ha & $\%$ & \\
\hline Argentina & 0 & 0 & 3892 & 24764 & 0 & 0 & 28656 & 0,78 & 4 \\
\hline Belice & 0 & 95800 & 0 & 0 & 0 & 0 & 95800 & 2,61 & 1 \\
\hline Bolivia & 0 & 803986 & 0 & 0 & 0 & 0 & 803986 & 21,91 & 8 \\
\hline Brasil & 4795 & 357464 & 34554 & 836325 & 4800 & 44000 & 1281938 & 34,93 & 30 \\
\hline Chile & 3588 & 0 & 13020 & 336969 & 0 & 0 & 353577 & 9,63 & 11 \\
\hline Colombia & 0 & 0 & 0 & 20056 & 0 & 0 & 20056 & 0,55 & 1 \\
\hline Costa Rica & 8975 & 0 & 21440 & 33621 & 0 & 0 & 64036 & 1,74 & 14 \\
\hline Ecuador & 1341 & 0 & 0 & 20000 & 0 & 0 & 21341 & 0,58 & 2 \\
\hline Guatemala & 0 & 64869 & 2242 & 0 & 28203 & 252808 & 348122 & 9,49 & 13 \\
\hline Honduras & 0 & 0 & 0 & 0 & 13398 & 0 & 13398 & 0,37 & 2 \\
\hline México & 18839 & 13748 & 0 & 0 & 84492 & 434106 & 551185 & 15,02 & 30 \\
\hline Nicaragua & 3500 & 0 & 0 & 0 & 0 & 0 & 3500 & 0,10 & 1 \\
\hline Panamá & 0 & 0 & 9099 & 0 & 0 & 0 & 9099 & 0,25 & 4 \\
\hline Uruguay & 0 & 0 & 5040 & 70023 & 0 & 0 & 75063 & 2,05 & 4 \\
\hline Total & 41038 & 1335867 & 89287 & 1341758 & 130893 & 730914 & 3669757 & 100 & 125 \\
\hline$\%$ & 1,12 & 36,40 & 2,43 & 36,56 & 3,57 & 19,92 & 100 & & \\
\hline
\end{tabular}

nco: Número de certificados otorgados

Fuente: FSC, Abril de 2003. In: http://www.fscoax.org.

baja y se corre el riesgo de que la producción se destine a mercados convencionales; así, el costo se convierte en una barrera para los pequeños propietarios y medianas empresas (van Dam, 2002).

Simula (2001) señala que de convertirse la certificación en un requisito básico para el mercado internacional, la diferencia de precio que pudiera existir probablemente desaparecerá, lo cual traería un efecto inverso al planteado, con el riesgo de inducir la tala ilegal y el cambio de uso del suelo (Prabhu et al., 1998; van Dam, 2002).
En la práctica, se carece de un mercado justo para la madera certificada, que compense la inversión que hace el productor para manejar su bosque de manera sustentable. El mercado actual de los productos forestales certificados es marginal; la certificación como herramienta de mercado se comporta como un costo fijo que contribuye únicamente a promover el MFS en bosques productivos y plantaciones comerciales muy rentables y con fácil acceso al mercado (van Dam, 2002).

La certificación forestal no contribuye al desarrollo socioeconómico, ni a la 
disminución de la deforestación ni a la restauración de bosques degradados situados en ecosistemas pobres con especies forestales de bajo valor para el mercado y en manos de pequeños productores y comunidades campesinas 0 indígenas, situación característica de las zonas forestales de México. Por lo tanto, la certificación es potencialmente útil tan solo para una superficie reducida de bosques naturales, resolviendo parcialmente el problema para lo que fue creada. Además, no es compatible con el desarrollo forestal comunitario pues, en éste, con el aprovechamiento y comercialización de la mayor cantidad y volumen posible de productos se contribuye a resolver la problemática de pobreza, degradación y deforestación.

Actualmente, la demanda de madera certificada aumenta pero concentrándose en países ambientalmente concientes de Europa Occidental (Reino Unido, países bajos y Alemania) y América del Norte. Está compuesta básicamente por redes de comercio forestal y entidades públicas (Kiekens, 1995; Savenije, 1999; Aguirre et al., 2000; FAO, 2001). Por su parte, la oferta es baja y limitada, con inconsistencia en la calidad, deficiente flujo industrial y bajo rendimiento.

El comercio de la madera certificada tiene poca importancia a nivel mundial; ningún país ha certificado más del $5 \%$ de su producción maderable (van Dam, 2002). En promedio, internacionalmente se comercializa un volumen de 1,7 millones de $\mathrm{m}^{3}$ de madera certificada lo que representa el $3 \%$ del total de la producción mundial (FAO, 2001; Rice et al., 2001). Por lo tanto, la contribución de la certificación a la conservación de los bosques es muy modesta.

Existe poca evidencia que permita establecer que la madera certificada obtendría un sobreprecio en el mercado, no obstante, algunos sectores del mercado están dispuestos a pagar un sobreprecio desde un $5 \%$ hasta un $14 \%$ con relación al precio de la madera no certificada, pero su disposición disminuye conforme aumenta el precio del producto (Baharuddin, 1995; Ozanne y Vlosky, 1997; BID, 2002). Otros sectores, en cambio, modificarían su patrón de consumo en caso de haber incremento, ya que, en general, los consumidores seleccionan los productos de madera más con base en un precio bajo y mejor calidad que en factores ambientales (Salim et al., 1997).

\section{DESVENTAJAS Y LIMITACIONES DE LA CERTIFICACIÓN}

Aún cuando es el Estado quien norma y regula la gestión, el manejo y el aprovechamiento de los bosques a través de la legislación forestal y políticas públicas sectoriales, las certificadoras minimizan su participación por lo que sus lineamientos para el MFS no tienen carácter legal, situación que es percibida por los productores como una duplicación de esfuerzos, con funciones paralelas y disociadas (Simula, 2001; van Dam, 2002).

La proliferación de sistemas globales de certificación, muchas veces contradictorios, representa un problema para los productores ya que para satisfacer distintos mercados se ven obligados a obtener más de un certificado para un mismo producto o bien volver a la forma de manejo convencional (Carbarle y de Freitas, 1995; Baharuddin, 1995; Rametsteiner, 2001) caracterizado por que el aprovechamiento es selectivo, ineficiente, de corto plazo e irresponsable social y ambientalmente.

Para solventar el caso anterior es importante establecer como denominador común un marco para la armonización nacional e internacional y acuerdos de reconocimiento mutuo; además es necesario la creación de estándares nacionales que se apeguen a las condiciones económicas, sociales y ecológicas específicas para cada región. 
Por otra parte, la certificación sólo evalúa el MFS tomando como empresa a la UMF de interés y no se centra en la región o territorio (van Dam, 2002), por lo que no cambia la forma de operación del modelo convencional de desarrollo y los procesos de deforestación y degradación continúan. La situación empeora cuando la superficie a certificar es reducida, debido a que entonces los efectos favorables que se pretenden sobre la biodiversidad y la conservación a nivel del paisaje ecológico no son cubiertos (Barthod, 1998; Tickell, 2000), ya que las UMF no siguen demarcaciones naturales y los procesos de interés rebasan sus límites. Esto también hace imposible monitorear los efectos de la fragmentación en los procesos biofísicos y socioeconómicos que determinan la estructura del paisaje y la forma en que cambia el uso del suelo (Prabhu et al., 1998; Pierce y Ervin, 1999; Herrera y Corrales, 1999).

Además de lo antes indicado, dado que las sucesiones bióticas son integraciones de procesos biológicos, químicos y físicos que requieren años o siglos para manifestarse (Jeffers, 1996; Rondeux, 1999), una evaluación técnica para otorgar la certificación no es suficiente para garantizar la sustentabilidad puesto que no permite realizar comparaciones en espacio y tiempo.

Finalmente, la certificación carece de estándares aplicables a los productos forestales no maderables (PFNM) y a las externalidades que benefician a la sociedad por lo que subestima el valor real de los bosques (ACDI, 2000; Tickell, 2000; Simula, 2001) desalentado el MFS y las inversiones necesarias a largo plazo. Los bosques deben ser valiosos para sus propietarios y los beneficios distribuidos equitativamente de tal forma que les brinden un retorno justo derivado de su aprovechamiento, de lo contrario, la experiencia ha demostrado que mientras los bosques no tengan un valor para la población local seguirán siendo destruidos o convertidos a otros usos del suelo.

\section{ALTERNATIVAS PARA CONTRIBUIR AL MFS}

Una situación característica altamente cuestionable de la certificación es que hasta ahora considera para el mercado una sola función del bosque: la madera; los bienes y servicios ambientales como conservación de la biodiversidad y el paisaje, regulación del clima, captura de carbono, mejora en la calidad del aire, captación de agua, protección del suelo y PFNM, no son valorados y menos aún incluidos como productos para el mercado (van Dam, 2002).

El hecho de que sean exclusivamente los productores quienes internalicen los costos sin obtener remuneración por los conceptos indicados y que los beneficios los reciba toda la sociedad, representa un obstáculo que impide o limita el buen desarrollo y aceptación de la certificación (Baharuddin, 1995; Simula, 2001). Para contribuir a aliviar esta situación, es necesario que se desarrollen en forma paralela al de la madera nuevos esquemas de certificación específicos para incluir en el mercado nacional o internacional a los bienes y servicios ambientales ya referidos. La creación de instrumentos y desarrollo de proyectos sobre usos forestales no consuntivos como el desarrollo de actividades recreativas, safaris fotográficos y ecoturismo representan alternativas para avanzar hacia el MFS.

Además, el MFS puede ser más factible mediante la utilización del bosque en forma diversificada e integral con objetivos múltiples mediante: a) aprovechamiento tanto de productos maderables como no maderables, estos últimos tienen potencial para entrar al comercio internacional generando ingresos y beneficios socioeconómicos en el ámbito local donde otras opciones son limitadas; b). silvicultura comunitaria y; c). agroforestería que puede contribuir a tratar con el problema de la escasez de leña al emplearse árboles de uso múltiple. 
En todos los casos indicados, siempre debe preverse que el índice de cosecha y la capacidad instalada no deben exceder la capacidad de suministro de los bosques. La sola cosecha de los mejores árboles para ser vendidos a los mercados de exportación como madera sin procesar es una práctica que debe rebasarse.

\section{CONCLUSIONES}

La certificación forestal como mecanismo de mercado hasta ahora, ha fortalecido exclusivamente a un mercado de madera dominado por grandes productores y empresas que si bien no mejoran sus precios, forman un oligopolio para adueñarse de un mercado selectivo y excluyente. Carece de incentivos para pequeños propietarios y comunidades que no tienen ni la capacidad empresarial ni el capital necesarios para alcanzar el manejo exigido, situación que los hace poco competitivos en comparación con los países desarrollados y con quienes hacen un manejo no sustentable, además de que su madera la destinan a mercados locales, regionales o nacionales donde no existe certificación.

La certificación será viable cuando el mercado origine una prima de precio para la madera certificada; de esta manera, los pequeños productores que están al margen de la economía global podrían inclinarse por la certificación, pasando del manejo convencional al sustentable, fomentando así la cantidad y calidad de los bosques y mejorando el desempeño, la competitividad y la compatibilidad internacional.

La certificación es una herramienta complementaria a otros instrumentos y políticas nacionales e internacionales que también promueven el MFS, constituye una solución parcial y una alternativa adicional para promover y monitorear el MFS.
El desarrollo total de la certificación no será una condición suficiente para alcanzar el MFS, no es una panacea que resuelve los problemas de deforestación y degradación forestal y tiene todavía un largo camino por delante.

El éxito y la eficacia de la certificación forestal dependerán de factores como el desarrollo de los mercados, la inclusión de los costos de certificación y los de mejoramiento del manejo en los precios de los productos. Otros factores de éxito de la certificación son las políticas forestales, el papel de los gobiernos, las reglas de la comercialización, la credibilidad, la armonización y el mutuo reconocimiento de los sistemas de certificación.

\section{REFERENCIAS}

ACDI (Agencia Canadiense para el Desarrollo Internacional). 2000. Asuntos forestales: Los bosques tropicales y el medio ambiente. Dirección General de Políticas. Québec, Canadá. 9 p.

Aguirre, J.; C. Blackburn; M. Mieres; C. Miquel y M. Henry. 2000. Manual instructivo sobre certificación forestal. Instituto Forestal. Grupo de Medio Ambiente. Santiago, Chile. 19 p.

Baharuddin, H.G. 1995. Timber certification: an overview. Unasylva 46(183):18-24.

Barthod, C. 1998. Criteria and indicators for sustainable temperate forest management - 1992 to 1996. Unasylva 49(192):53-56.

BID (Banco Interamericano de Desarrollo). 2002. Estrategia regional de biodiversidad para los países del trópico Andino. La Paz, Bolivia. 72 p. 
Cabarle, B. y A.R. de Freitas. 1995. Timber certification and the pursuit of credible claims. Unasylva 46(183):25-26.

Castilleja, G. 1993. Cambios en las políticas forestales de América Latina: Los casos de Chile, Nicaragua y México. Unasylva Vol. 44(175):23-31.

De Camino, R. y M. Alfaro. 2000. Certification in Latin America - experience to date. In: Swedish University of Agriculture Sciences (eds): Forests, trees and people newsletter No. 43. Uppsala, Suecia. p:25-27.

FAO. 2001. State of the World's Forests 2001. Roma, Italia. $181 \mathrm{p}$.

FAO. 2003. State of the World's Forests 2003. Roma, Italia. $151 \mathrm{p}$.

Herrera, B. y L. Corrales.1999. Propuesta metodológica para la selección de criterios e indicadores y análisis de verificadores relativos a calidad de bosque y a nivel de paisaje. Documento para discusión. Unión Mundial para la Naturaleza (UICN). Oficina Regional para Meso América (ORMA). Área de Conservación de Bosques y Áreas Protegidas (ACBAP). San José, Costa Rica. 16 p.

Jeffers J., N.R. 1996. Measurement and characterization of biodiversity in forest ecosystems. New methods and models. European Forest Institute, EFI Proceedings 6:59-67.

Kiekens, J.P. 1995. Timber Certification: a critique. Unasylva 46(183):27-28.

Ozanne, L.K. y R.P. Vlosky. 1997. Willingness to pay for environmentally certified wood products: A Consumer Perspective. Forest Products Journal. 47(6):39-48.

Pierce, R.A. y J. Ervin B. 1999. Can independent forest management certification incorporate elements of landscape ecology. Unasylva 50(196):49-56.

Prabhu R.; C. Colfer y G. Shepherd. 1998. Criterios e indicadores para la ordenación forestal sostenible: Nuevos hallazgos de la investigación realizada por CIFOR al nivel de la unidad de manejo forestal. Red Forestal para el Desarrollo Rural. Documento RDFN número 23a. 20 p.

Rametsteiner, E. 2001. Pasado, presente y futuro: certificación forestal. Ambiente y Desarrollo 27(1):30-40.

Rice, R.E.; C.A. Sugal, S.M. Ratay y G.A. Fonseca. 2001. Manejo forestal sostenible: Revisión del saber convencional. Advances in Applied Biodiversity Science, No. 3. Washington, DC: CABS/Conservation International. $35 \mathrm{p}$.

Rondeux, J. 1999. Forest inventories and biodiversity. Unasylva 50(196):35-41.

Salim, E.; U. Djalins U. y A. Suntana. 1997. Comercio y certificación de productos forestales: un esquema Indonesio. In: Comercio de productos forestales y certificación. No. 22. FAO. Sección Montes. p:135-144.

Sasser, E.N. 2002. Gaining leverage: NGO influence on certification institutions in the forest products sector. In: Teeter, L.; B. Cashore y D. Zhang (eds). Forest policy for private forestry. CAB International. pp:229244.

Savenije J., F.H. 1999. Manejo sostenible de los bosques y la certificación voluntaria: desarrollos internacionales y perspectivas para el sector forestal y maderero en Perú. In: II Encuentro nacional maderero. Pucallpa, Perú. 20-23 de Junio 1999. $21 \mathrm{p}$. 
Simula, M. 2001. Comercio y medio ambiente en la producción forestal. Banco Interamericano de Desarrollo. Departamento de Desarrollo Sostenible. División de Medio Ambiente. Washington, D.C. 38 p.

Tickell, O. 2000. Certificación: un futuro para los bosques del mundo. World Wildlife Fund. $37 \mathrm{p}$.

Upton, C. y S. Bass 1995. The forest certification handbook. Earthscan
Publications Ltd. Londres, Reino Unido. 219 p.

van Dam, C. 2002. La economía de la certificación forestal: ¿desarrollo sostenible para quién?. Universidad Nacional de Salta, Argentina. Ponencia a presentarse en el Congreso Iberoamericano de Desarrollo y Medio Ambiente: Desafíos locales ante la globalización. Noviembre de 2002. Quito, Ecuador. 25 p. $\diamond$

1 INIFAP CIR-Centro. Campo Experimental "San Martinito". Km 56.5 carretera federal México-Puebla. Tlahuapan 74100 Pue. c.e.: cesmar@compu-redes.net.mx.

Manuscrito recibido el 23 de junio de 2003.

Aceptado el 5 de septiembre de2003.

Este documento se debe citar como:

Tamarit U., J.C. 2003. Análisis del escenario de la certificación forestal en el contexto del desarrollo sustentable. Madera y Bosques 9(2):3-13. 